\title{
Enabling Smart Home Networks with ZigBee Technology
}

\author{
Siquan $\mathrm{Hu}^{1, \mathrm{a}^{*}}$, He Zhou ${ }^{1, \mathrm{~b}}$ and Chundong She ${ }^{2, \mathrm{c}}$ \\ ${ }^{1}$ School of Computer and Communication Engineering, University of Science and Technology \\ Beijing, Beijing, 100083, China \\ ${ }^{2}$ School of Electronic Engineering, Beijing University of Posts and Telecommunications, Beijing, \\ 100876, China \\ ahusiquan@ustb.edu.cn, b664884497@qq.com, cshurcd@vip.sina.com
}

Keywords: Smart Home Network, ZigBee, CC2530

Abstract. ZigBee technology is a short range, low-cost, low power-consumption and low data rate wireless sensor network technology, which makes it a suitable technology for smart home networks. In this paper we present a systematic method to construct smart home networks with ZigBee chip. Both hardware design and software design are presented. The node hardware uses core ZigBee board to provide ZigBee communication support for the specific nodes. The designs of both a smart socket node and another central control and display node are presented. The association software flow of mapping from the control key on the control node to in-net smart socket power relay is also illustrated. Deployment test showed the validation of our design.

\section{Introduction}

Smart home networks provide a comfortable, safe, high quality and pleasant family living space. Compared to traditional home networks, the in-progress ubiquitous home network collects user activity awareness, as well as physical sensing information in the home environment, to support more smart and well-being home services. It has the essential to easy control consumer home network services used in livelihood. Eventually, users will experience the convenience of performing ordinary life styles and increased satisfaction offered by adaptive home services

Currently many researches are carried out on enabling smart home networks. Most work focus on building a Smart Home Energy Management System to save energy. [1, 2]To make smart home devices more energy efficient and smart, Jinsung Byun et al. proposed a situation based self-adjusting scheme, and an event-based self-adjusting sensor network based on ZigBee sensor networks [3]. Smart home network has some specific characteristics comparing with traditional networks such as noise electromagnetic noise and context-aware [4]. ZigBee technology is a short range, low-cost, low power-consumption and low data rate wireless sensor network technology, which makes it a suitable technology for smart home networks [5]. In this paper we present a systematic method to construct smart home networks with ZigBee chip. Both hardware design and software design are presented.

\section{Hardware Design}

Core ZigBee Board Design. In our design, a smart home network consists of many ZigBee nodes. The ZigBee nodes may vary much on their functions such as controlling the consumer devices or sampling the sensors' data or a centre control point or a display node. Since all nodes should have the ZigBee communication capabilities, we propose a core ZigBee board to share the design over all the different nodes. CC2530 is selected as the ZigBee chip in this design. CC2530 is a true system-on-chip (SoC) solution for IEEE 802.15.4, ZigBee and RF4CE applications. CC2530 combines the excellent performance of a leading RF transceiver with an industry-standard enhanced $8051 \mathrm{MCU}$, in-system programmable flash memory, 8-KB RAM, and many other powerful features. Fig.1 shows the PCB design on the left and the final product on the right. The core board use a stamp structure, with most GPIO are routed to the board outline. The RF part is self-contained with an on-board $2.4 \mathrm{GHz}$ antenna to save the extra effort of RF redesign on each specific node. 


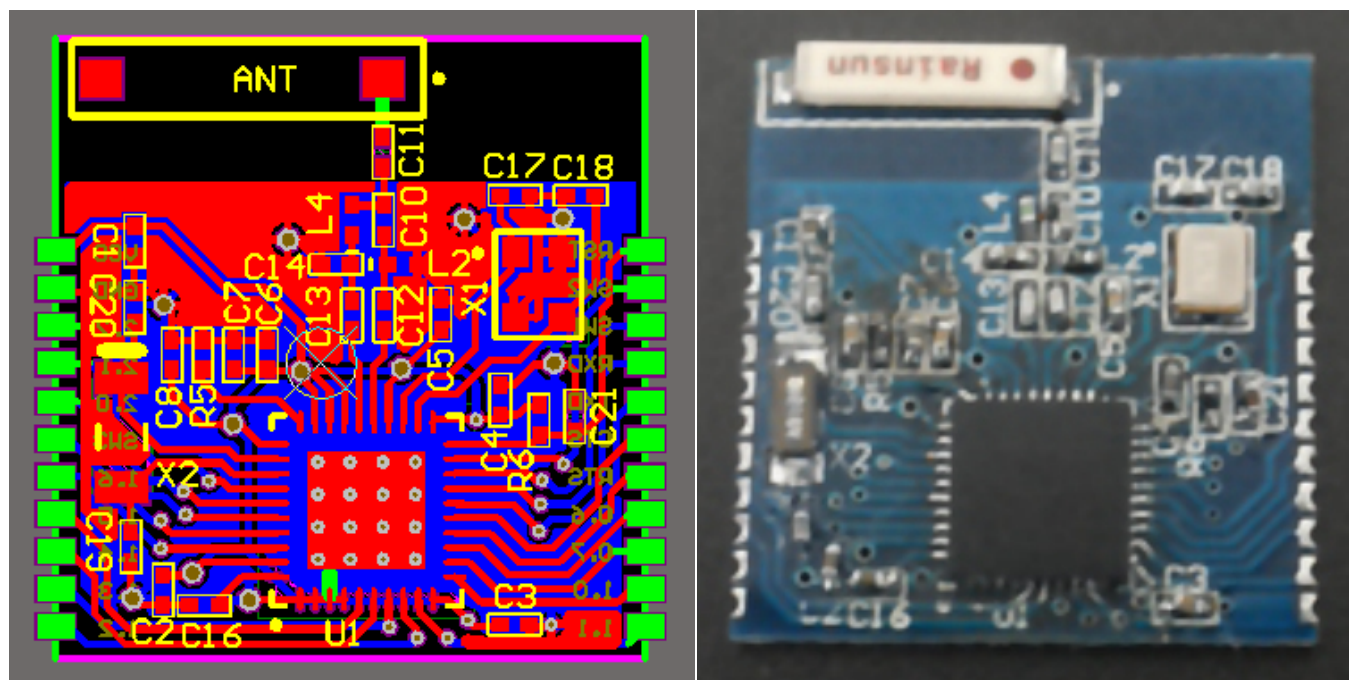

Fig. 1 The core ZigBee .

Smart Socket Node Design. A smart home network may have device interface nodes to interact with TV or refrigerator etc. It also may have node to sample the sensor data such as temperature or humidity. We present a sample node smart socket with both power relay to control the ON/OFF of the power plug and a temperature and humidity sensor DHT11 to get the sensor data. Fig. 2 shows the schematic and real product of the smart socket board. The power is source from wall AC and a $220 \mathrm{~V}-3.3 \mathrm{~V}$ converter is used. The core Zigbee board is used as the core module to provide the ZigBee wireless communication ability.

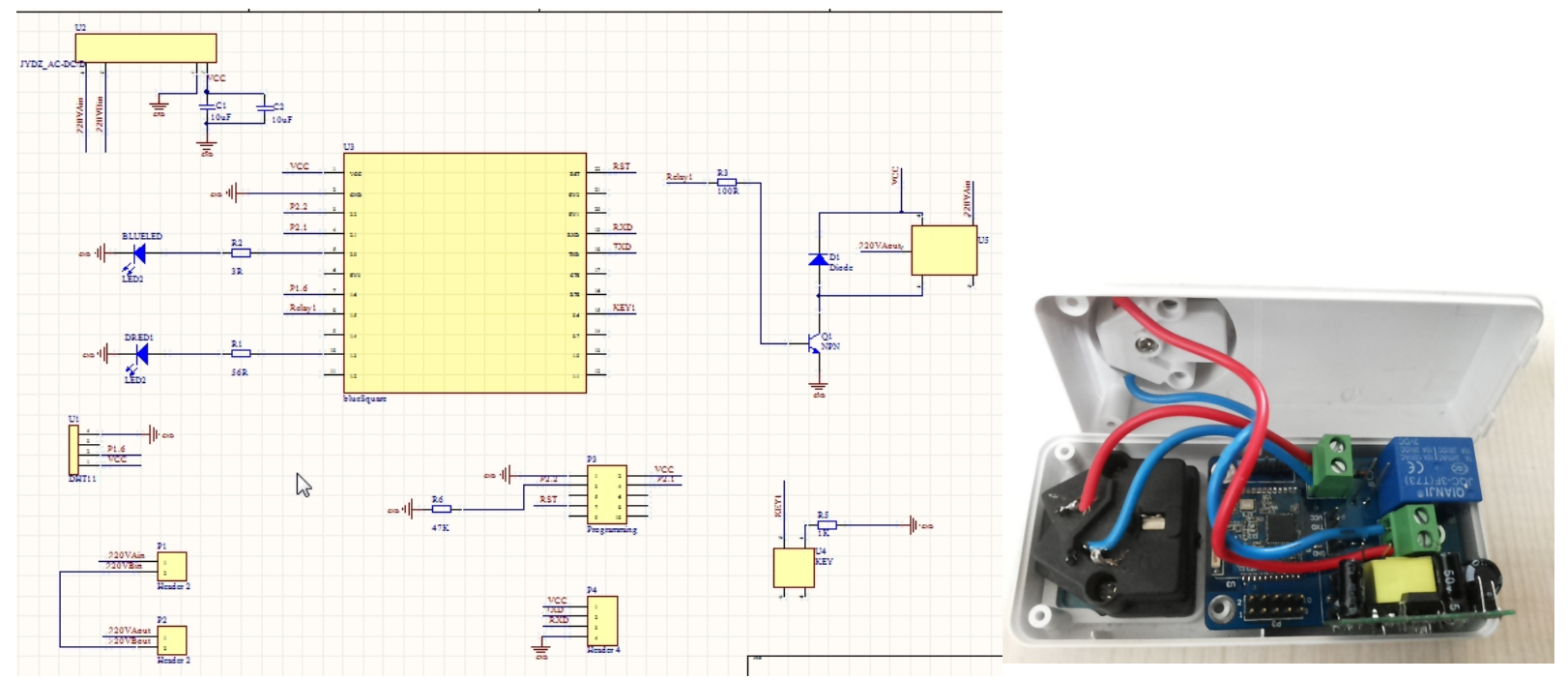

Fig. 2 A smart socket node in a smart home network.

Control and Display Node Design. A smart home network may have a central control node to control the consumer devices and a display node to show the data of the in-network nodes. In our design, a compound control and display node is used to demonstrate the control node and display node design method. Fig. 3 shows the schematic and real product of the control and display node. The four keys are used to turn on or off the power of the associated smart sockets. A LCD1602 LCD display is used to show the temperature and humidity data of in-net smart socket nodes. The software will 


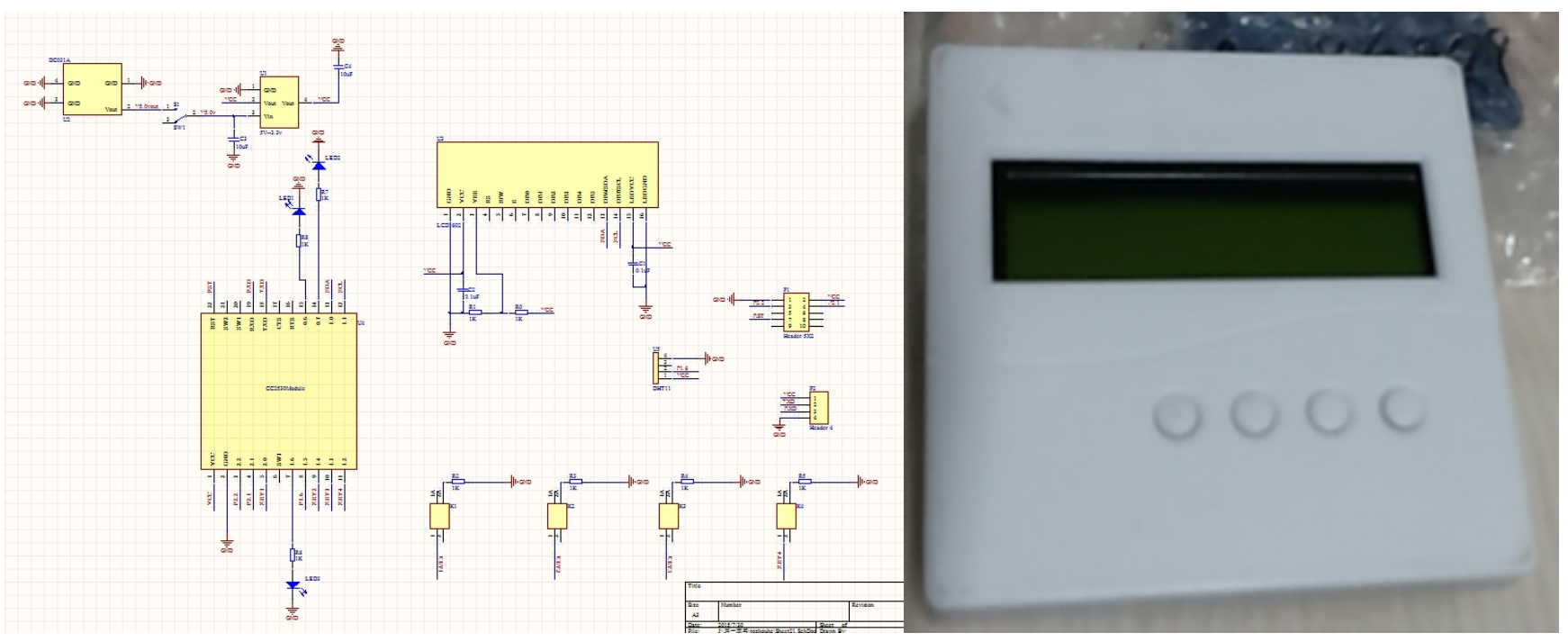

Fig. 3 A control and display node in smart home network.

\section{Software Design}

With the help of TI stack ZigBee 2007, we can design the firmware of both the smart socket node and the control node. ZigBee use a simple event loop operating system OSAL. To map the control key from the control node to the power relay switch of the smart socket nodes, we use the event map provided by the Zigbee 2007. We define a key event for each key and map the key event of key to an endpoint of the control node. When started, first we should bind the power relay of the smart socket $n$ to the endpoint $\mathrm{n}$ of the control node. Then the control node can send the Toggle command to the smart socket node with no need to know the short address of the smart socket node.

Since in a ZigBee network we can't get the network address fixed before network set up, above mapping will be a little tricky. We can use binding to conquer it. First, there is a key on the smart socket $\mathrm{n}$. Pushing this key can set the smart socket on the binding- allowing status. Then pushing the key $\mathrm{n}$ of the control node will initiate a binding request to power relay endpoint of the smart socket node. If both of the key actions are within a short time, the binding process will succeed. Since the key $n$ is mapped to the endpoint $\mathrm{n}$ on control node, then the endpoint $\mathrm{n}$ is bind to power relay of the smart socket. After binding, any time the key $\mathrm{n}$ on the control node is pushed, the $z b \_S e n d D a t a R e q u e s t$ function will be called to send the Toggle command to the bonded smart socket with a invalid destination address as the parameter. Since the key $n$ is used as a bind request key on the configuration stage but use a power relay command later, a status will be used to track the binding process. After binding, the key will be used to control the corresponding smart socket only; following code will be called at such time.

\{

// send the command to toggle the power relay

Zb_SendDataRequest( 0xFFEE, TOGGLE_POWER_RELAY_CMD_ID, 0, (uint8 *)NULL, myAppSeqNumber, 0, 0);

\section{System Test}

To validate our design, we use four smart socket nodes and one control and display node to form a simple smart home network as in Figure 4. The Control and Display node is served as the coordinator of the Zigbee network. All smart socket node s are configured as ZigBee routers. Each smart socket has a lamp bulb to show the effect of remote turn on/off from the control node. All the sensor data re 
displayed on the LCD1602 display. Real test show both control path and the sensor data gathering are successful.

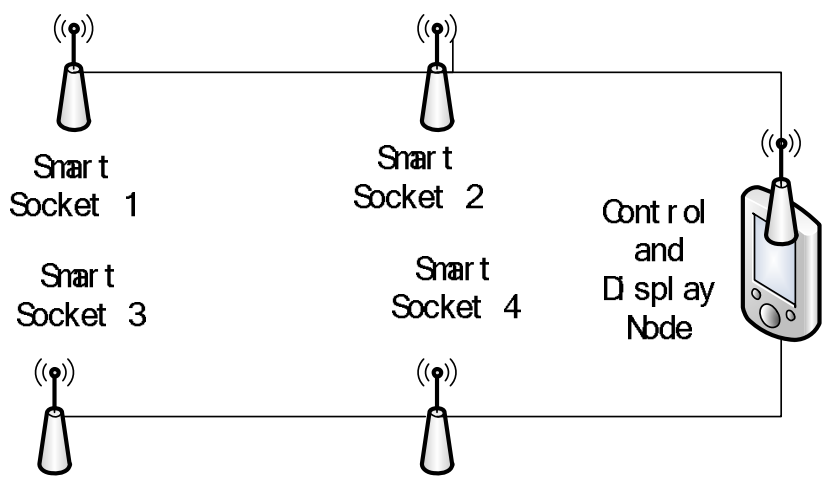

Fig. 4 The test smart home network.

\section{Conclusion}

In this paper we present a systematic method to construct smart home networks with ZigBee chip. Both hardware design and software design are illustrated. The deployment test showed the validation of our design. Future work will expand the scale of the network and interact with more concrete consumer devices.

\section{Acknowledgements}

The study was supported by the National Natural Science Foundation of China (No. 91438120) and the Foundation of Key Laboratory of Aerospace Broadband Network Technology.

\section{References}

[1] Jinsoo H, Chang-sic C, Wan-Ki P, et al. Smart home energy management system including renewable energy based on ZigBee and PLC. IEEE Trans. on Consumer Electronics. Vol.60(2), 2014: 198-202.

[2] Dae-Man H, Jae-Hyun L. Design and implementation of smart home energy management systems based on Zigbee. IEEE Trans. on Consumer Electronics. Vol.56(3), 2010: 1417-1425.

[3] JinSung B, Boungju J, Junyoung N, et al. An intelligent self-adjusting sensor for smart home services based on ZigBee communications[J]. IEEE Trans. on Consumer Electronics. Vol.58(3), 2012: 794-802.

[4] Idoudi M, Elkhorchani H, Grayaa K. Performance evaluation of Wireless Sensor Networks based on ZigBee technology in smart home. ICEESA 2013. 2013:1-4.

[5] Zhen B. Smart home with ZigBee hardware simulation and performance evaluation. International Conference on Mechatronic Sciences, Electric Engineering and Computer. IEEE,2013:2139-2142. 